\title{
Leaching Effect on Multiple Surfaces of a Single Grain Decagonal Al-Ni-Co Quasicrystal
}

\author{
M. Lowe ${ }^{a, *}$, P. Gille ${ }^{b}$, R. MCGRath ${ }^{a}$ And H.R. Sharma ${ }^{a}$ \\ ${ }^{a}$ Surface Science Research Centre, University of Liverpool, Liverpool, L69 3BX, UK \\ ${ }^{b}$ Department of Earth and Environmental Sciences, Crystallography Section \\ Ludwig-Maximilians-Universität München, Germany
}

\begin{abstract}
We have studied the effect of leaching treatments on the surface microstructure and chemical composition on the two different twofold surfaces, (10000) and (10100), and the tenfold surface (00001) of a decagonal (d-) $\mathrm{Al}-\mathrm{Ni}-\mathrm{Co}$ quasicrystal. A sample exposing the three surfaces was leached with $\mathrm{NaOH}$ solution and then studied using atomic force microscopy and scanning electron microscopy combined with energy dispersive X-ray analysis. The leaching treatment preferentially removes the $\mathrm{Al}$ present in the crystal surface leaving a mixture of transition metal and their associated oxides. The high symmetry, tenfold axis was found to be less resistant to leaching than either of the twofold axes, providing further information regarding the effect of crystallographic direction on leaching kinetic.
\end{abstract}

DOI: 10.12693 /APhysPolA.126.520

PACS: 61.44.Br, 68.37.Hk

\section{Introduction}

In previous work, it has been documented that $\mathrm{Al}$ based quasicrystals can be altered through chemical etching using $\mathrm{NaOH}$, for the production of catalysts [1-3]. The surface Al was removed through leaching to leave a surface covered in transition metal particles. In the case of $\mathrm{Al}-\mathrm{Cu}-\mathrm{Fe}$, a surface consisting of $\mathrm{Cu}$ and $\mathrm{Fe}$ particles was observed. It was also found that samples of similar stoichiometry exhibited different leaching rates that could be attributed to their crystal structure, crystalline materials being the most aggressively leached, while the quasicrystalline materials were the most resistant [4]. A result that suggested this was part of a trend rather than a single outcome was the finding that the quasicrystal approximants displayed a leaching resistance greater than crystalline samples but less than quasicrystal samples [3]. Despite previous work, there is to date no clear understanding of the interaction between the quasicrystalline bulk and the leached layer [5].

In our previous studies we observed that leaching of the fivefold surface of the icosahedral $\mathrm{Al}-\mathrm{Cu}-\mathrm{Fe}$ produced fivefold facets upon the surface [6]. On the leached crystal there were places where pentagonal and pentagonal-dodecahedral cavities were found on macroscopic surface orientations that were no longer well-aligned to the original fivefold surface. Similar results have previously been observed during oxidation studies on the Al-Pd-Mn family quasicrystals where agressive treatments unearthed other "internal" surface orientations $[7,8]$. This indicated a preference for the leaching of the fivefold direction. The pentagonal-dodecahedral cavities also demonstrated the

*corresponding author; e-mail: mikelowe@liverpool.ac.uk leaching of other fivefold surfaces at angles of $108^{\circ}$. This led to the initial conclusion that the fivefold crystallographic direction was prefered to the others, regardless of which macroscopic surface was exposed to the leaching solution.

In a bid to test this hypothesis further and directly, we used a single crystal decagonal $\mathrm{Al}-\mathrm{Ni}-\mathrm{Co}$ quasicrystal with 3 well orientated surfaces present on the sample. Decagonal quasicrystals are characterised by the periodic stacking of quasicrystalline planes [9]. The high symmetry axes of these systems includes a unique tenfold axis along the periodic direction and two inequivalent twofold axes. These twofold planes appear alternately at $18^{\circ}$ to each other and perpendicular to the tenfold axis. The twofold planes therefore comprise of both the periodic and quasiperiodic direction. This allows a direct comparison of the leaching kinetics. An additional advantage over the previously studied powdered samples is the "simplified" nature of the single crystal surfaces. This paper will outline the experiments undertaken to compare the leaching characteristics of 3 surfaces on the $\mathrm{d}-\mathrm{Al}-\mathrm{Ni}-\mathrm{Co}$ quasicrystal, two crystalline surfaces (00001) and (10100), and the 2D quasicrystalline plane (00001). Scanning electron microscopy (SEM) and energy dispersive X-ray analysis (EDX) were used to investigate both chemical and structural effects of the leaching treatment.

\section{Experimental details}

A decagonal $\mathrm{Al}-\mathrm{Ni}-\mathrm{Co}$ quasicrystal was cast into a cuboid with polished (00001), (10000) and (10100) surfaces, see Fig. 1. Figure 1a shows the sample "as-grown" with labelled surfaces, while Fig. 1b shows the relationship of the surface orientations on our sample with those in the decagonal structure of the crystal. 


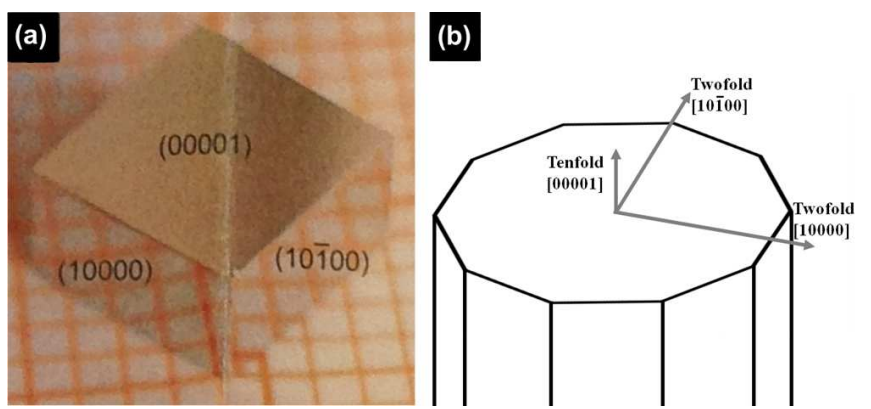

Fig. 1. (a) Crystal as-grown and polished. (b) Layout of the surfaces on a decagonal rod.

The surfaces were mechanically polished using diamond paste with diameter $5-0.25 \mu \mathrm{m}$. The crystal was then suspended in 5 mol concentration $\mathrm{NaOH}$ solution and leached for $5 \mathrm{~min}$ in ambient conditions. The surfaces were then investigated using SEM, EDX, and atomic force microscopy (AFM).

\section{Results and discussion}

\subsection{The twofold (10000) surface}

Figure 2 shows a summary of the SEM data for the (10000) twofold surface. The surface is characterised mostly as very flat, with a slight puckering of the surface, which can be seen in Fig. 2b. This can be attributed in places to the presence of many small circular depressions often with at least a single crack forming through the middle. These can be seen in detail in Fig. $2 \mathrm{c}$ and $\mathrm{d}$. The symmetry of these features is different from the surface symmetry, indicating that any order present from the twofold surface has been lost. As is common in previous Al-based quasicrystal leaching experiments, the surface is coated in a homogeneous precipitate particle layer of transition metal.

\subsection{The twofold (10100) surface}

With minimum zoom using the SEM the dramatic effect of leaching can be seen in the (10100) surface (Fig. 3). Surprisingly, compared to the previous twofold surface, there is the creation of ordered rows of leached grooves that stretch for long distances across the crystal and examples from two different parts of the surface can be seen in Fig. 3b and d. These lines are almost parallel, often with slight convergence around areas of leached cavities or at the edges of the crystal. Most lines are separated either by $\approx 6 \mu \mathrm{m}$ or $\approx 12 \mu \mathrm{m}( \pm 0.3 \mu \mathrm{m})$.

The lines are distinct from those caused by polishing by their near parallel nature, scale and by the observance of polishing grooves crossing the leached lines. What makes these straight grooves of even more note is their orientation with respect to the rest of the crystal. The grooves run perpendicular to the tenfold surface and continue for several hundred $\mu \mathrm{m}$ across the surface.

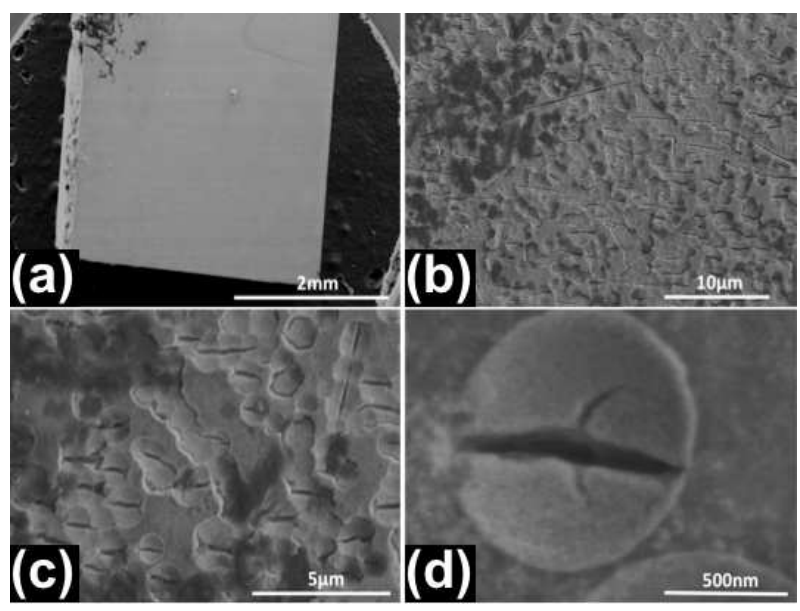

Fig. 2. SEM images of the (10000) surface following leaching. (a) Wide zoom of the surface. (b) Characteristic roughness of the surface observed. (c) Features of the roughness. (d) Example of repeating facet on the surface.

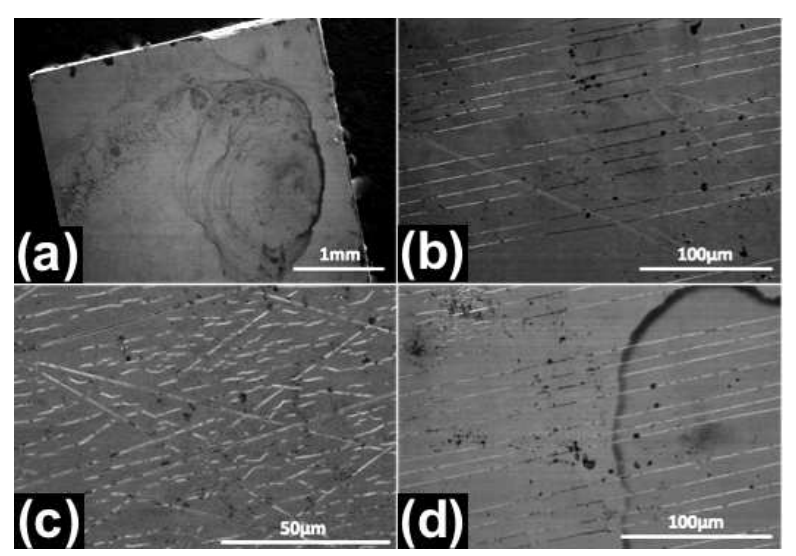

Fig. 3. Surface of the leached (10100) surface. (a) Wide zoom of leached surface. (b) Lines aligned perpendicular to the (00001) tenfold surface. (c) Areas of non parallel features are still common across the surface. (d) Similar lines and line separations from a distant part of the crystal can be directly compared with that in part (b).

The work of Mäder et al. demonstrated a clean twofold surface was characterised by a columnar structure perpendicular to the tenfold surface [10]. The terminations of these columns were observed to be parallel to the tenfold surface. The columnar structure observed on the clean surface and the leaching induced grooves observed in our work are therefore both orientated along the periodic axis. The width of the grooves is significantly larger than the columnar structures they report. We therefore suggest that the parallel trenches observed on the etched surface are formed during leaching, where the exposed columnar structures on the clean surface act as nucleation sites. 


\subsection{The tenfold (00001) surface}

The leached surface of the (00001) orientation can be seen, even optically, to be more leached than the other surfaces previously mentioned. The surface is very dark and much darker than the other two surfaces. This was different to previous experiments with i-Al- $\mathrm{Cu}-\mathrm{Fe}$ where similar results would require several hours of leaching. This indicates a preference for leaching along the tenfold direction, and when considered with previous results, a preference for leaching along the periodic direction. In comparison the greatly increased leaching resistance of the $\mathrm{i}-\mathrm{Al}-\mathrm{Cu}-\mathrm{Fe}$ system can be considered to be due to the icosahedral structure, which provides quasiperiodic ordering in all directions, as opposed to the decagonal structure, which has periodic directions. The surface remains mostly flat apart from the presence of similar troughs to the (10100) surface, although these are far less regularly patterned (Fig. 4). Figure 4c shows an example of using AFM to identify the depth of these grooves to be of the order of $\approx 100 \mathrm{~nm}$ and to be largely unchanged in depth at other points across the surface. We suggest that the unusual patterns on the surface displayed in Fig. 4c, and their inconsistency with the other planes can be attributed to the quasiperiodic nature of the surface, although no aperiodic order could be identified.

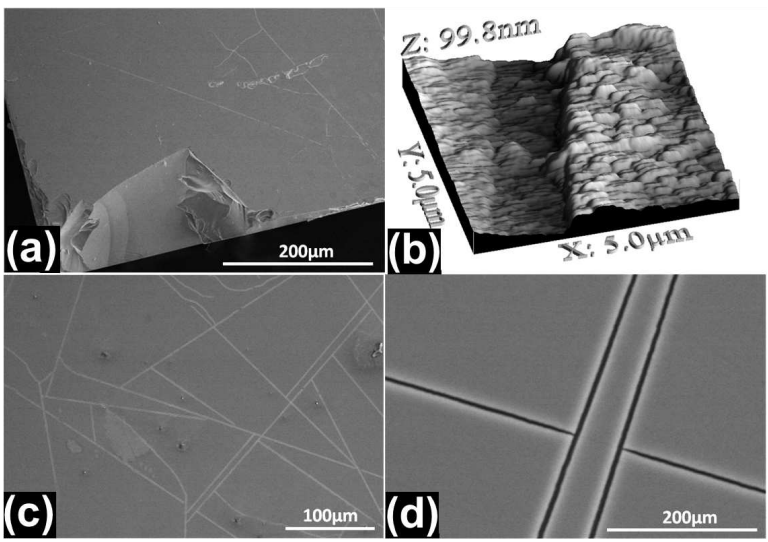

Fig. 4. (a) SEM image at wide zoom of the (00001) surface. (b) AFM image of etched grooves present on surface. (c) SEM image of the grooves produced across a large area. (d) Small scale image of connecting grooves.

\subsection{EDX comparison}

All the surfaces appear similarly chemically changed following leaching treatment as measured using EDX. This appears to suggest uniform leaching of all surfaces however, the low surface sensitivity of the technique means that small variations in response to leaching would not be recognised. EDX has an information depth of several $\mu \mathrm{m}$, we do not believe the leach depth to be on the same scale, based on previous single crystal leaching work using X-ray photoelectron spectroscopy (XPS) on $\mathrm{i}-\mathrm{Al}-\mathrm{Cu}-\mathrm{Fe}$. Therefore it is possible that the variation in leaching stability, from a chemical analysis technique, will not be visible with such a large proportion of the signal originating from the bulk. We can however use EDX to indicate changes in leach depth. We expect the surface to be Al free for at least the information depth of XPS (several atomic layers), and therefore any Al signal in EDX must come from the bulk. Therefore variation in this signal may indicate the change in depth of the leached layer.

The EDX results show a reduction in $\mathrm{Al}$ in all observed surfaces, as would be expected based upon previous work. Coupled with this is the observed increase in transition metals and oxygen. The increase in oxygen is attributed largely, but not exclusively, to the $\mathrm{Cu}$ and $\mathrm{Ni}$ oxide species which are formed during leaching. EDX is not capable of determining oxide species in the manner of other spectroscopic techniques such as XPS. Figure 5 shows that there is a consistent increase in $\mathrm{Co}$ and $\mathrm{Ni}$ on all three surfaces. The ratio of Co to Ni remains approximately constant during this treatment, indicating there is no preferential selection of either during the leaching process.

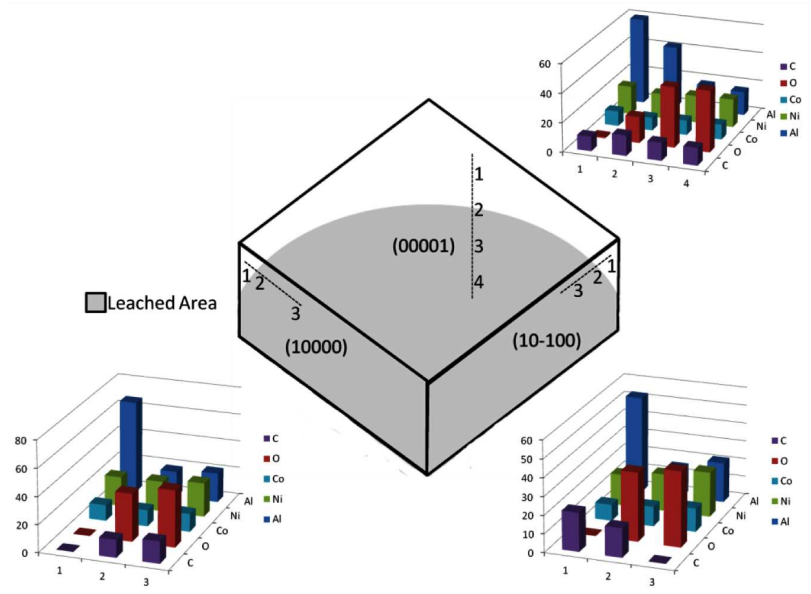

Fig. 5. Comparison of chemical composition (at.\%) variation across leaching boundaries, or transition regions, on all three Al-Ni-Co surfaces (00001), (10000), and $(10 \overline{1} 00)$. Numbers represent points across the surface at which spectra were taken. The dark grey area represents the leached area of the crystal.

There is a difference in the chemical characteristics of the leaching boundary between the twofold surfaces and the tenfold surface. In the twofold surfaces, the Al content changes dramatically upon entering the leaching boundary and then changes very little even once totally inside the leached region. This can be seen on both (10000) and (10100) surfaces in Fig. 5, where the Al content decreases from point 1 to point 2 . We have interpreted this to be caused by the proposed resistance to leaching along quasiperiodic directions, which causes the transition region to be small and to avoid leaching parallel to the surface.

In the tenfold surface, the $\mathrm{Al}$ content changes steadily across the leaching boundary and into the entirely 
leached region. This leaching boundary is difficult to distinguish in the tenfold surface using SEM, whereas in the twofold surfaces it was a clear band running across the crystal. This indicates a continuous increase in leach depth across the crystal. This is interpreted to be due to an increased leaching kinetic along the tenfold direction. The leaching is still present along the other directions but the speed of this is reduced leading to the continuous slope and a poorly defined leaching boundary.

\section{Conclusions}

Three orientated surfaces of a decagonal Al-Ni-Co quasicrystal were leached in $\mathrm{NaOH}$ and then studied using SEM and EDX. Both twofold surfaces were found to be less aggressively leached than the quasiperiodic tenfold surface. Leached microstructure indicates preferential leaching along the periodic directions, again indicating that crystallographic direction rather than macroscopic surface orientation is the key influence on leaching kinetics of quasicrystals. This gives further weight to the idea that the quasiperiodic arrangement of $\mathrm{Al}$ in the crystal inhibits alkali leaching.

\section{Acknowledgments}

The authors are grateful to R. Clowes and A.I. Cooper for the use of high resolution SEM within the University of Liverpool Centre for Materials Discovery. The authors would also like to thank E. Aoyagi and A.P. Tsai from Tohoku University for technical expertise and helpful discussions. The authors also thank D. Martin from the University of Liverpool for providing AFM. M.L. would like to acknowledge the support of the EPSRC doctoral training grant and the European Integrated Centre for the Development of New Metallic Alloys and Compounds (C-MAC) for scientific exchange funding.

\section{References}

[1] A.P. Tsai, M. Yoshimura, Appl. Catal. A, Gen. 214, 237 (2001).

[2] A.P. Tsai, M. Yoshimura, Mater. Res. Soc. Symp. Proc. 643, K16.4.1 (2001).

[3] T. Tanabe, S. Kameoka, A.P. Tsai, Catal. Today 111, 153 (2006).

[4] T. Tanabe, S. Kameoka, A.P. Tsai, Appl. Catal. A, Gen. 384, 241 (2010).

[5] S. Kameoka, T. Tanabe, A.P. Tsai, Catal. Today 93-95, 23 (2004).

[6] T.P. Yadav, M. Lowe, R. Tamura, R. McGrath, H.R. Sharma, in: Aperiodic Crystals, Eds. S. Schmid, R.L. Withers, R. Lifshitz, Springer, Netherlands 2013, p. 275.

[7] C. Beeli, T. Gödecke, R. Lück, Philos. Mag. Lett. 78, 339 (1998).

[8] D. Rouxel, M. Gil-Gavatz, P. Pigeat, B. Weber, J. Non-Cryst. Solids 351, 802 (2005).

[9] H.R. Sharma, M. Shimoda, A.P. Tsai, Adv. Phys. 56, 403 (2007).

[10] R. Mäder, R. Widmer, P. Gröning, S. Deloudi, W. Steurer, M. Heggen, P. Schall, M. Feuerbacher, O. Gröning, Phys. Rev. B 80, 035433 (2009). 